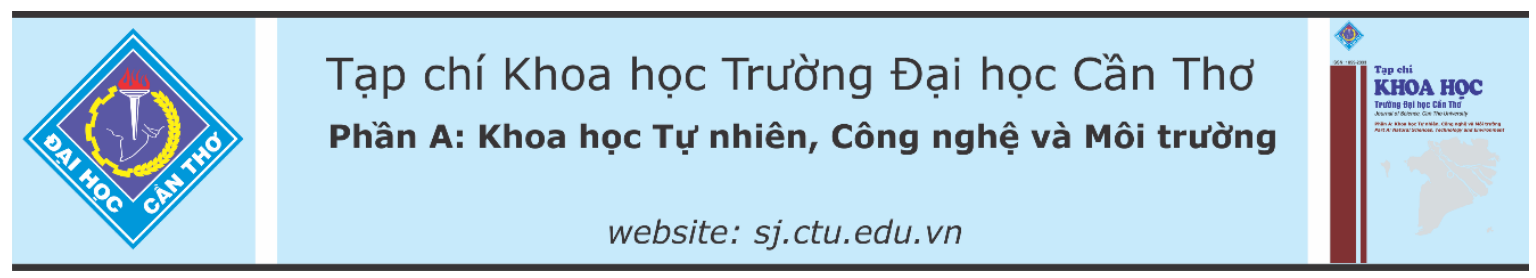

DOI:10.22144/ctu.jvn.2021.145

\title{
SỰ TỒN TẠI VÀ TÍNH NỬA LIÊN TỤC TRÊN CỦA NGHIÊM BÀI TOÁN CÂN BẰNG VECTOR THEO NÓN THỨ TỰ CÓ PHẦN TRONG ĐẠI SỐ KHÁC RỖNG
}

Trần Ngọc Tâm ${ }^{1^{*}}$, Hà Nguyễn Huỳnh $\mathrm{Anh}^{2}$, Trần Thị Kim $\mathrm{Anh}^{2}$, Dương Minh Nhật ${ }^{2}$ và Nguyễn Ngọc Minh Thy ${ }^{2}$

${ }^{1}$ Bộ môn Toán, Khoa Khoa học Tự nhiên, Trường Đại học Cần Tho

${ }^{2}$ Ngành Su phạm Toán hoc, Khoa Su phạm, Truòng Đại hoc Cần Tho

*Nguời chịu trách nhiệm về bài viết: Trần Ngoc Tâm (email: tntam@ctu.edu.vn)

\section{Thông tin chung:}

Ngày nhận bài: 10/04/2021

Ngày nhận bài sủa: 01/07/2021

Ngày duyệt đăng: 29/10/2021

\section{Title:}

Existence and upper semicontinuity of solutions to equilibrium problems via an ordering cone with nonempty algebraic interior

\section{Tù khóa:}

Bài toán cân bằng vector, phần trong đại số, tính nửa liên tục trên

Keywords:

Algebraic interior, upper

semicontinuity, vector equilibrium problem

\section{GIỚI THIÊU}

Bài toán cân bằng vector được giới thiệu trong các công trình của Ansari et al., 1997; Oettli, 1997 và Bianchi et al., 1997. Trong hơn hai thập kỷ qua, bài toán cân bằng vector đã được nghiên cứu một cách rộng rãi, bởi vì bài toán này chứa rất nhiều bài toán quan trọng trong tối ưu như bài toán tối ưu vector, bài toán bất đẳng thức biến phân vector, bài toán điểm yên ngựa vector, bài toán về điểm bất động, bài toán bù và bài toán cân bằng Nash vector (Ansari, 2000). Cũng như các lớp bài toán khác, sự tồn tại nghiệm của bài toán cân bằng vector luôn là chủ đề nhận được nhiều sự quan tâm của các nhà toán học. Đã có rất nhiều công trình nghiên cứu về chủ đề sự tồn tại nghiệm của bài toán cân bằng được công bố (Ansari et al., 2001; Ansari, 2008; Bigi et al., 2010; Chen et al., 2005; Gong, 2006; Huang et al., 2007). Chủ đề quan trọng tiếp theo của sự tồn tại nghiệm là tính ổn định nghiệm. Đây là một chủ đề được quan tâm trong thời gian gần đây nhưng tốc độ phát triển rất nhanh. Tính ổn định nghiệm ở đây có thể được hiểu ở hai dạng chính. Dạng thứ nhất là tính nửa liên tục hoặc liên tục theo nghĩa Hausdorff và Berge của ánh xạ nghiệm (Anh et al., 2019, 2020, 2021). Dạng thứ hai là tính liên tục theo nghĩa Hölder hoặc Lipschitz của ánh xạ nghiệm (Anh et al., 2018; Tam, 2021). 
Một điều đáng lưu ý là các kết quả về sự tồn tại nghiệm và tính ổn định nghiệm của bài toán cân bằng vector đạt được ở các công trình trên chủ yếu dựa vào các giả thiết về tính lồi của hàm mục tiêu hoặc thông qua các công cụ đòi hỏi phải có tính chất topo của không gian ảnh đối với hàm mục tiêu. Mục tiêu của bài báo là nghiên cứu sự tồn tại và tính nửa liên tục trên của nghiệm cho bài toán cân bằng vector theo nón thứ tự có phần trong đại số khác rỗng. Nghĩa là bài toán được xét với không gian ảnh không đòi hỏi phải có bất kỳ một topo đặc biệt nào cũng như không đòi hỏi tính lồi của hàm mục tiêu. Một cách cụ thể hơn, bài báo thiết lập các điều kiện đủ cho tính khác rỗng và tính nửa liên tục trên của nghiệm bài toán cân bằng vector với không gian ảnh là không gian tuyến tính thực thông qua các công cụ về nửa liên tục trên theo nón và tính lồi giảm nhẹ của các tập mức (yếu hơn tính lồi của hàm mục tiêu).

\section{KIẾN THỨC CHUẨN BI!}

Từ phần này trở về sau, nếu không giả thiết gì thêm, xét $X$ là một không gian vector topo Hausdorff và $Y$ là một không gian tuyến tính thực.

\section{Định nghĩa 2.1}

Tập $\Omega \subset Y$ được gọi là một tập lồi nếu với mọi $x, y \in \Omega$ và $t \in[0,1]$ thì

$$
t x+(1-t) y \in \Omega \text {. }
$$
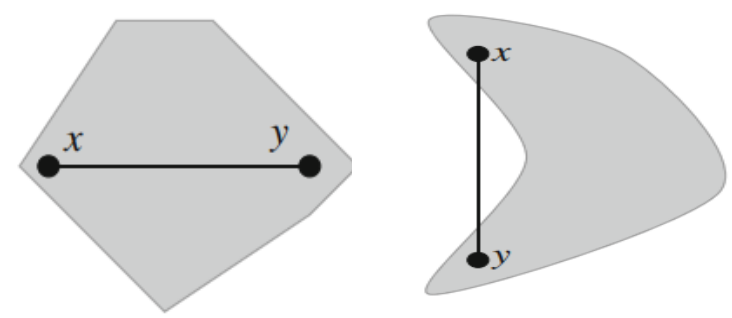

\section{Hình 1. Tập lồi (trái) và tập không lồi (phải)}

Định nghĩa 2.2 (Phần trong đại số của một tập)

Cho $\Omega$ là một tập con khác rỗng của không gian tuyến tính $Y$. Phần trong đại số của tập $\Omega$, kí hiệu là $\operatorname{core}(\Omega)$, được định nghĩa như sau:

$$
\begin{gathered}
\operatorname{core}(\Omega):=\{a \in \Omega: \forall v \in Y, \exists \delta> \\
0 \text { sao cho } a+[0, \delta] v \subset \Omega\} .
\end{gathered}
$$

Tập $\Omega$ thỏa mãn điều kiện $\operatorname{core}(\Omega)=\Omega$ được gọi là một tập mơ đại số.

Nếu $Y$ là một không gian vector topo thì về mặt tổng quát ta có:

$$
\operatorname{int}(\Omega) \subseteq \operatorname{core}(\Omega),
$$

trong đó int $(\Omega)$ là kí hiệu phần trong topo của $\Omega$. Ta xét ví dụ sau:

\section{Ví dụ 2.1}

Cho $Y=\mathbb{R}^{2}$ và tập $\Omega$ được xác định như sau:

$$
\Omega:=\left\{\left(y_{1}, y_{2}\right): y_{2} \geq y_{1}^{2} \text { hoặc } y_{2} \leq 0\right\} \text {. }
$$

Khi đó, $\quad \operatorname{core}(\Omega)=\left\{\left(y_{1}, y_{2}\right): y_{2}>\right.$ $y_{1}^{2}$ hoặc $\left.y_{2}<0\right\} \cup\{\mathbf{0}\}$ (phần gạch như trong Hình 2).

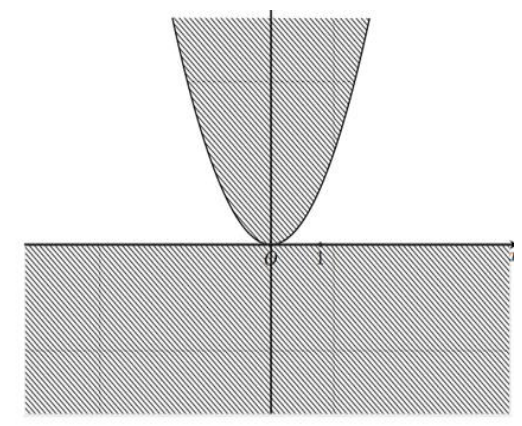

Hình 2.

Trong khi đó, phần trong topo của $\Omega$ là int $(\Omega)=$ $\left\{\left(y_{1}, y_{2}\right): y_{2}>y_{1}^{2}\right.$ hoặc $\left.y_{2}<0\right\}$. Do đó, $\operatorname{int}(\Omega) \subset$ $\operatorname{core}(\Omega)$.

Mệnh đề 2.1 (Kantorovitz, 2003, Bổ đề 5.17, Tr. 134)

Nếu $\Omega$ là một tập lồi và có phần trong topo khác rỗng trong không gian topo tuyến tính $Y$ thì $\operatorname{int}(\Omega)=\operatorname{core}(\Omega)$.

Mệnh đề 2.2 (Aliprantis \& Border, 2007, Bổ đề 5.60, Tr. 200)

Nếu $\Omega$ là một tập lồi khác rỗng trong không gian topo tuyến tính hữu hạn chiều $Y$ thì $\operatorname{int}(\Omega)=$ $\operatorname{core}(\Omega)$.

Mệnh đề 2.3 (Bonnans \& Shapiro, 2000, Chú ý 2.73, Tr. 56)

Nếu $\Omega$ là một tập lồi đóng trong không gian metric tuyến tính đầy đủ $Y$ ( $Y$ là một không gian metric đầy đủ được trang bị một cấu trúc tuyến tính sao cho các phép toán cộng và phép nhân với vô hướng là liên tục) thì int $(\Omega)=\operatorname{core}(\Omega)$.

Sau đó, một số tính chất của phần trong đại số của một tập con trong không gian tuyến tính thực được xét.

\section{Mệnh đề 2.4}

Cho $\Omega$ là tập con lồi trong $Y$. Khi đó: 
a) $\operatorname{core}(\Omega)$ là một tập lồi.

b) Nếu $a \in \operatorname{core}(\Omega)$ và $b \in \Omega$ thì $[a, b) \subset$ $\operatorname{core}(\Omega)$ với

$$
[a, b)=\{\theta a+(1-\theta) b, 0<\theta \leq 1\} .
$$

\section{Chứng minh:}

a) Giả sử $a, b \in \operatorname{core}(\Omega)$ và $0 \leq \lambda \leq 1$. Theo định nghĩa của phần trong đại số thì với bất kỳ $v \in$ $Y$, tồn tại $\delta>0$ sao cho $a+t v \in \Omega$ và $b+t v \in \Omega$ với $t \in[0, \delta]$.

Do tính lồi của $\Omega$ nên với mỗi $t$ ta có

$$
\begin{aligned}
\lambda a+(1-\lambda) b+ & t v \\
& =\lambda(a+t v)+(1-\lambda)(b+t v) \\
& \in \lambda \Omega+(1-\lambda) \Omega \subset \Omega .
\end{aligned}
$$

Điều này có nghĩa là $\lambda a+(1-\lambda) b \in \operatorname{core}(\Omega)$ và do đó core $(\Omega)$ là một tập lồi.

b) Lấy $\lambda \in(0,1]$ và đặt $y_{\lambda}:=\lambda a+(1-\lambda) b$. Ta sẽ chứng minh $y_{\lambda} \in \operatorname{core}(\Omega)$. Vì $a \in \operatorname{core}(\Omega)$ nên với mỗi $v \in Y$ thì tồn tại $\delta>0$ sao cho

$a+t v \in \Omega$ với $t \in[0, \delta]$.

Sử dụng tính lồi của $\Omega$, ta được

$$
\begin{aligned}
y_{\lambda}+t \lambda v=\lambda a+ & (1-\lambda) b+t \lambda v \\
& =\lambda(a+t v)+(1-\lambda) b \\
& \in \lambda \Omega+(1-\lambda) \Omega \subset \Omega,
\end{aligned}
$$

tức là $y_{\lambda} \in \operatorname{core}(\Omega)$.

\section{Mệnh đề 2.5}

Cho $\Omega \subset Y$ và $y_{0} \in \Omega$. Giả sử rằng với mọi $v \in$ $Y$ thì tồn tại $\delta>0$ với $0<\lambda<\delta$ sao cho $y_{0}+\lambda v \in$ $\Omega$. Khi đó $y_{0} \in \operatorname{core}(\Omega)$.

\section{Chứng minh:}

Với mọi $v \in Y$, ta chọn $\delta_{0}=\frac{\delta}{2}$ thì khi đó $\delta_{0}>$ 0 và $y_{0}+\lambda v \in \Omega$ với mọi $\lambda \in\left[0, \delta_{0}\right]$. Điều này chứng tỏ $y_{0} \in \operatorname{core}(\Omega)$.

\section{Mệnh đề 2.6}

Cho $\Omega$ là tập con lồi của $Y$. Khi đó $\operatorname{core}(\Omega)$ là một tập mở đại số trong $Y$.

\section{Chứng minh:}

Để chứng minh $\operatorname{core}(\Omega)$ là một tập mở đại số trong $Y$ ta sẽ chứng minh $\operatorname{core}(\operatorname{core}(\Omega))=$ $\operatorname{core}(\Omega)$.

Vì $\Omega$ là tập lồi nên theo Mệnh đề 2.4 a) ta suy $\operatorname{core}(\Omega)$ cũng là một tập lồi. Do đó, $\operatorname{core}(\operatorname{core}(\Omega))$ cũng là một tập lồi. Vì $\operatorname{core}(\Omega) \subset \Omega$ nên $\operatorname{core}(\operatorname{core}(\Omega)) \subset \operatorname{core}(\Omega)$.

Để chứng minh điều ngược lại, ta lấy $a \in$ $\operatorname{core}(\Omega)$ và lấy $v \in Y$. Theo định nghĩa của phần trong đại số thì tồn tại $\delta>0$ sao cho

$a+t v \in \Omega$ với $t \in[0, \delta]$,

và do đó $a+\frac{\delta}{2} v \in \Omega$. Với mọi $\gamma$ thỏa mãn $0<$ $\gamma<\frac{\delta}{2}$, ta xét số $\lambda$ được định nghĩa bởi

$$
\lambda:=1-\frac{2 \gamma}{\delta} .
$$

Khi $0<\gamma<\frac{\delta}{2}$, thấy $\lambda \in(0,1)$, nên theo Mệnh đề 2.4 thì $a+\gamma v=\lambda a+(1-\lambda)\left(a+\frac{\delta}{2} v\right) \in$ $\operatorname{core}(\Omega)$ với mọi $0<\gamma<\frac{\delta}{2}$.

Sử dụng kết quả của Mệnh đề 2.5, ta được $a \in$ $\operatorname{core}(\operatorname{core}(\Omega))$.

\section{Mệnh đề 2.7}

Cho $\Omega$ là tập con của $Y$. Khi đó, với bất kỳ tập $a \in Y$, ta có đẳng thức

$$
\operatorname{core}(a+\Omega)=a+\operatorname{core}(\Omega) \text {. }
$$

\section{Chứng minh:}

Lấy bất kỳ $x \in \operatorname{core}(\Omega)$, khi đó với mọi $v \in Y$ thì tồn tại $\lambda>0$ sao cho $x+t v \in \Omega$ với mọi $t \in$ $[0, \lambda]$. Suy ra $a+x+t v \in a+\Omega$. Do đó, $a+x \in$ $\operatorname{core}(a+\Omega)$. Vậy $a+\operatorname{core}(\Omega) \subset \operatorname{core}(a+\Omega)$.

Ngược lại, lấy bất kỳ $w \in \operatorname{core}(a+\Omega)$, khi đó với $v \in Y$ thì tồn tại $\lambda>0$ sao cho $w+t v \in a+\Omega$ với mọi $t \in[0, \lambda]$. Nếu $w \notin a+\operatorname{core}(\Omega)$ thì $w-$ $a \notin \operatorname{core}(\Omega)$. Do đó, tồn tại $v_{0} \in Y$ sao cho với mọi $\lambda_{0}>0$ thì tồn tại $t_{0} \in\left[0, \lambda_{0}\right]$ thỏa mãn $w-a+$ $t_{0} v_{0} \notin \Omega$ hay $w+t_{0} v_{0} \notin a+\Omega$. Suy ra $w \notin$ $\operatorname{core}(a+\Omega)$. Đây là một mâu thuẫn. Vậy core $(a+$ $\Omega) \subset a+\operatorname{core}(\Omega)$.

\section{Mệnh đề 2.8}

Nếu $\Omega$ là một tập con lồi và mở đại số trong $Y$ thì bất kỳ tập $A \subset Y$, ta có đẳng thức

$$
\operatorname{core}(A+\Omega)=A+\Omega \text {. }
$$

\section{Chứng minh:}

Hiển nhiên ta có: $\operatorname{core}(A+\Omega) \subset A+\Omega$.

Mặt khác: 


$$
\begin{gathered}
A+\Omega=\bigcup_{a \in A}(a+\Omega)=\bigcup_{a \in A}(a+\operatorname{core}(\Omega)) \\
=\bigcup_{\substack{a \in A \\
\subset \operatorname{core}(A+\Omega) .}}(\operatorname{core}(a+\Omega))
\end{gathered}
$$

Vậy $\operatorname{core}(A+\Omega)=A+\Omega$.

\section{Định nghĩa 2.3}

Cho $C$ là một tập con của $Y$. Khi đó:

(a) $C$ được gọi là nón nếu $\lambda x \in C$ với mọi $x \in C$ và $\lambda \geq 0$.

(b) Nón $C$ được gọi là có đỉnh nếu $C \cap(-C)=$ $\left\{\mathbf{0}_{Y}\right\}$, ở đây $\mathbf{0}_{Y}$ là vector không trong $Y$. lồi.

(c) Nón $C$ được gọi là nón lồi nếu $C$ là một tập
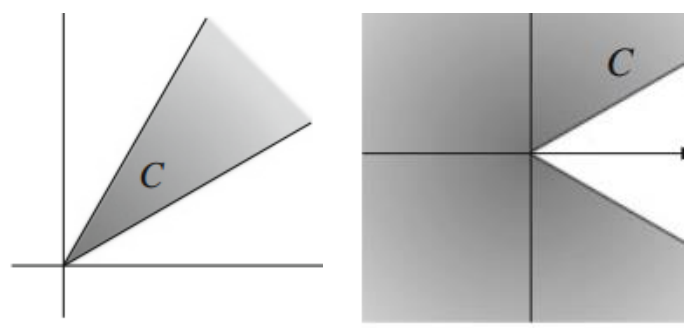

\section{Hình 3. Nón lồi (trái) và nón không lồi (phải)}

\section{Mệnh đề 2.9}

Nón $C$ là lồi trong $Y$ nếu và chỉ nếu $C+C \subset C$.

\section{Chứng minh:} thì

Giả sử $C$ là một nón lồi. Khi đó với mọi $x, y \in C$

$$
\frac{1}{2}(x+y)=\frac{1}{2} x+\frac{1}{2} y \in C,
$$

hay $x+y \in C$. Do đó, $C+C \subset C$.

Ngược lại, giả sử nón $C$ có tính chất $C+C \subset C$. Ta chứng minh $C$ là một tập lồi. Thật vậy, với mọi $x, y \in C$ và $t \in[0,1]$ thì $t x \in C$ và $(1-t) y \in C$. Suy ra, $t x+(1-t) y \in C+C \subset C$. Vậy $C$ là một tập lồi.

\section{Mệnh đề 2.10}

Nếu $C$ là một nón lồi trong $Y$ và $\operatorname{core}(C)$ khác rỗng thì core $(C)+C=\operatorname{core}(C)$.

\section{Chứng minh:}

Ta có: $\quad \operatorname{core}(C)=\left\{\mathbf{0}_{Y}\right\}+\operatorname{core}(C) \subset C+$ $\operatorname{core}(C)$. Do đó, bao hàm thức " $C$ " đã được chứng minh.

Với bao hàm thức " $\supset$ ", lấy bất kỳ $c_{1} \in C, c_{2} \in$ $\operatorname{core}(C)$ và $y \in Y$ thì tồn tại $\lambda>0$ sao cho

$c_{2}+t y \in C$ với mọi $t \in[0, \lambda]$.

Vì $C$ là nón lồi nên theo Mệnh đề 2.9 ta được

$c_{1}+c_{2}+t y \in C$ với mọi $t \in[0, \lambda]$.

Do đó $c_{1}+c_{2} \in \operatorname{core}(C)$. Vậy $C+\operatorname{core}(C) \subset$ $\operatorname{core}(C)$.

Cho $C$ là một nón lồi có đỉnh và core $(C)$ khác rỗng trong $Y$. Khi đó ta dùng các kí hiệu sau đây để chỉ quan hệ thứ tự được sinh ra bởi nón $C$. Với mọi $x, y \in Y$ :

$$
\begin{gathered}
x \geq y \Leftrightarrow x-y \in C, \\
x>y \Leftrightarrow x-y \in \operatorname{core}(C), \\
x \geq y \Leftrightarrow x-y \notin C, \\
x \ngtr y \Leftrightarrow x-y \notin \operatorname{core}(C),
\end{gathered}
$$

và tương tự cho các quan hệ $\leq,<, \Varangle, \nless, \ldots$

Khái niệm nửa liên tục trên theo nón thứ tự trong không gian tuyến tính thực $Y$ được giới thiệu trong định nghĩa sau.

\section{Định nghĩa 2.4}

Cho $h: X \rightarrow Y$ là một ánh xạ có giá trị vector. Ánh xạ $h$ được gọi là $C$-nửa liển tục trên tại $x_{0} \in$ $X$ nếu với mỗi tập $V$ mở đại số trong $Y$ thỏa mãn $h\left(x_{0}\right) \in V$ thì tồn tại một lân cận $U$ của $x_{0}$ sao cho $h(x) \in V-C$ với mọi $x \in U$.

Ta nói rằng ánh xạ $h: X \rightarrow Y$ là $C$-nửa liên tục trên trên một tập nào đó nếu nó là $C$-nửa liên tục trên tại mọi điểm thuộc tập đó.

\section{Định lý 2.1}

Ánh xạ $h: X \rightarrow Y$ là $C$-nửa liên tục trên trên $X$ khi và chỉ khi với mọi $b \in Y$ thì $h^{-1}(b-\operatorname{core}(C))$ là một tập mở trong $X$.

\section{Chứng minh}

Giả sử $h$ là $C$-nửa liên tục trên $X$. Với mọi $b \in Y$ và $x_{0} \in h^{-1}(b-\operatorname{core}(C))$ thì ta suy ra $h\left(x_{0}\right) \in$ $b-\operatorname{core}(C)$. Khi đó tồn tại một lân cận $U$ của $x_{0}$ sao cho $h(x) \in b-\operatorname{core}(C)-C$ với mọi $x \in U$ hay $h(x) \in b-\operatorname{core}(C)$ với mọi $x \in U$ (vì $-\operatorname{core}(C)-C=-\operatorname{core}(C))$. Suy ra $x \in h^{-1}(b-$ $\operatorname{core}(C))$, điều này dẫn đến $x_{0} \in U \subset h^{-1}(b-$ 
core $(C))$. Do đó $h^{-1}(b-\operatorname{core}(C))$ là một tập mở với mọi $b \in Y$.

Ngược lại, giả sử $h^{-1}(b-\operatorname{core}(C))$ là một tập mở với mọi $b \in Y$. Ta chứng minh rằng $h$ là $C$-nửa liên tục trên tại mọi điểm $x_{0} \in X$. Thật vậy, gọi $V$ là một tập mở đại số bất kỳ trong $Y$ thoả mãn $h\left(x_{0}\right) \in$ $V$ và $e$ là một vector bất kỳ thuộc $\operatorname{core}(C)$. Khi đó tồn tại $\lambda>0$ sao cho $h\left(x_{0}\right)+\frac{\lambda}{2} e \in V$. Ta thấy rằng $x_{0} \in h^{-1}\left(h\left(x_{0}\right)+\frac{\lambda}{2} e-\operatorname{core}(C)\right)$ và $h^{-1}\left(h\left(x_{0}\right)+\frac{\lambda}{2} e-\operatorname{core}(C)\right)$ là một tập mở nên tồn tại một lân cận $U$ của $x_{0}$ sao cho

$$
x_{0} \in U \subset h^{-1}\left(h\left(x_{0}\right)+\frac{\lambda}{2} e-\operatorname{core}(C)\right) \text {, }
$$

hay với mọi $x \in U$ thì $x \in h^{-1}\left(h\left(x_{0}\right)+\frac{\lambda}{2} e-\right.$ $\operatorname{core}(C))$. Do đó $h(x) \in h\left(x_{0}\right)+\frac{\lambda}{2} e-\operatorname{core}(C) \subset$ $V-C$ với mọi $x \in U$. Vậy $h$ là $C$-nửa liên tục trên tại $x_{0}$.

\section{SỬ TỒN TẠI NGHIỆM CỦA BÀI TOÁN CÂN BĂNG VECTOR}

Trong phần này, cho $C$ là một nón lồi có đỉnh và core $(C)$ khác rỗng trong $Y$. Cho $K$ là một tập con khác rỗng của $X$ và $f: K \times K \rightarrow Y$ là một song hàm. Ta xét bài toán cân bằng vector sau đây:

(VEP) Tìm $\bar{x} \in K$ sao cho $f(\bar{x}, y) \notin-C \backslash$ $\left\{\mathbf{0}_{Y}\right\}$ với mọi $y \in K$.

\section{Định nghĩa 3.1}

Vector $x \in K$ được gọi là một nghiệm hữu hiệu yếu của bài toán (VEP) nếu

$$
f(\bar{x}, y) \notin-\operatorname{core}(C), \forall y \in K,
$$

hay

$$
f(\bar{x}, y) \nless \mathbf{0}_{Y}, \forall y \in K .
$$

Định nghĩa 3.2 (Ansari, 2000, Định nghĩa 3, Tr. 3)

Cho $\varnothing \neq G \subset X$ và một ánh xạ đa trị $F: G \rightrightarrows X$. Ánh xạ $F$ được gọi là một ánh xạ KKM nếu với mọi tập con hữu hạn $\left\{y_{1}, y_{2}, \ldots, y_{n}\right\}$ của $G$ thì quan hệ

$$
\operatorname{conv}\left\{y_{1}, y_{2}, \ldots, y_{n}\right\} \subset \bigcup_{i=1}^{n} F\left(y_{i}\right),
$$

được thỏa mãn, ở đây $\operatorname{conv}(E)$ là kí hiệu bao lồi của tập con $E$.

\section{Ví dụ 3.1}

Cho $G=X=\mathbb{R}$ và ánh xạ đa trị $F: G \rightrightarrows X$ được xác định bởi $F(y)=[0, y]$. Khi đó $F$ là một ánh xạ KKM.

Bổ đề sau thường được gọi là Bổ đề KKM-Fan.

Bổ đề 3.1 (Fan, 1961, Bổ đề 1)

Giả sử rằng $F: G \rightrightarrows X$ là một ánh xạ KKM có giá trị đóng. Khi đó, nếu tồn tại ít nhất một $\bar{y} \in G$ sao cho $F(\bar{y})$ là tập compact thì

$$
\bigcap_{y \in G} F(y) \neq \varnothing .
$$

Định lý sau đây phát biểu về sự tồn tại nghiệm yếu của bài toán (VEP).

\section{Định lý 3.1}

Giả sử rằng các điều kiện sau được thỏa mãn:

(i) với mọi $x \in K, f(x, x) \nless \mathbf{0}_{Y}$;

(ii) với mọi $x \in K$, tập $\left\{y \in K: f(x, y)<\mathbf{0}_{Y}\right\}$ là một tập lồi;

(iii) với mọi $y \in K, f(\cdot, y)$ là $C$-nửa liên tục trên trên $K$;

(iv) (điều kiện bức) tồn tại một tập compact khác rỗng $N$ của $K$ và $\bar{y} \in N$ sao cho $f(x, \bar{y})<\mathbf{0}_{Y}$ với mọi $x \in K \backslash N$.

Khi đó, tập nghiệm hữu hiệu yếu của bài toán (VEP) là khác rỗng.

\section{Chứng minh:}

Xét ánh xạ đa trị $F: K \rightrightarrows K$ được xác định bởi

$F(y):=\left\{x \in K: f(x, y) \nless \mathbf{0}_{Y}\right\}$ với mọi $y \in K$.

Ta thấy rằng, $\bar{x}$ là một nghiệm hữu hiệu yếu của bài toán (VEP) nếu và chỉ nếu

$$
\bar{x} \in \bigcap_{y \in K} F(y) .
$$

Trước hết, ta chứng minh $F$ là một ánh xạ KKM. Giả sử ngược lại, $F$ không là ánh xạ KKM thì khi đó tồn tại một tập con hữu hạn của $\left\{y_{1}, y_{2}, \ldots, y_{n}\right\}$ của $K$ và $y \in \operatorname{conv}\left\{y_{1}, y_{2}, \ldots, y_{n}\right\}$ nhưng $y \notin F\left(y_{i}\right)$ với mọi $i$. Do đó, $f\left(y, y_{i}\right)<\mathbf{0}_{Y}$ với mọi $i$. Vì $y \in$ conv $\left\{y_{1}, y_{2}, \ldots, y_{n}\right\}$ nên $y$ được viết dưới dạng $y=$ $\sum_{i=1}^{n} t_{i} y_{i}$ với $t_{i} \geq 0$ và $\sum_{i=1}^{n} t_{i}=1$.

Từ $f\left(y, y_{i}\right)<\mathbf{0}_{Y}$ với mọi $i$ và giả thiết (ii), ta suy ra 


$$
f\left(y, \sum_{i=1}^{n} t_{i} y_{i}\right)<\mathbf{0}_{Y} .
$$

Điều này tương đương với $f(y, y)<\mathbf{0}_{Y}$. Đây là điều không thể vì nó mâu thuẫn với giả thiết (i). Do đó, $F$ là một ánh xạ KKM.

Ta chứng minh $F(y)$ là một tập đóng trong $K$ với mọi $y \in K$. Thật vậy, lấy bất kỳ lưới $\left\{x_{\alpha}\right\} \subset F(y)$ với $x_{\alpha} \rightarrow x_{0}$. Nếu $x_{0} \notin F(y)$ thì $f\left(x_{0}, y\right)<\mathbf{0}_{Y}$ hay $f\left(x_{0}, y\right) \in-\operatorname{core}(C)$. Theo tính $C$-nửa liên tục trên của $f$ trong giả thiết (iii) thì tồn tại một lân cận $U$ của $x_{0}$ sao cho

$$
f(x, y) \in-\operatorname{core}(C)-C, \forall x \in U .
$$

Kết hợp điều này với Mệnh đề 2.10 ta suy ra $f(x, y) \in-\operatorname{core}(C)$ với mọi $x \in U$. Vì $U$ là một lân cận của $x_{0}$ và $x_{\alpha} \rightarrow x_{0}$ nên tồn tại $\alpha_{0}$ sao cho $x_{\alpha} \in$ $U$ với $\alpha \geq \alpha_{0}$. Khi đó $f\left(x_{\alpha}, y\right) \in-\operatorname{core}(C)$, đây là điều mâu thuẫn vì $x_{\alpha} \in F(y)$. Do đó, $x_{0} \in F(y)$ và ta suy ra $F(y)$ là một tập đóng trong $K$.

Với mỗi $\bar{x} \in F(\bar{y})$ bất kỳ thì ta có $f(\bar{x}, \bar{y}) \nless \mathbf{0}_{Y}$. Theo giả thiết (iv) thì $\bar{x} \notin K \backslash N$ hay $\bar{x} \in N$. Do đó, $F(\bar{y}) \subset N$. Hơn nữa, theo chứng minh trên thì $F(\bar{y})$ là một tập đóng. Vì $F(\bar{y})$ là một tập con đóng trong tập compact $N$ nên $F(\bar{y})$ cũng là một tập compact.

Như vậy, ta đã chứng minh được $F$ là một ánh xạ KKM với giá trị đóng và có $F(\bar{y})$ là một tập compact trong $K$. Áp dụng của bổ đề KKM-Fan, ta được:

$$
\bigcap_{y \in K} F(y) \neq \varnothing .
$$

Vậy tập nghiệm hữu hiệu yếu của bài toán (VEP) là khác rỗng.

\section{TÍNH NỬA LIÊN TUC TRÊN CỦA ÁNH XA NGHIÊM}

Mục này nghiên cứu bài toán (VEP) khi hàm mục tiêu và tập ràng buộc bị nhiễu bởi tham số $\lambda \in$ $\Lambda$ với $\Lambda$ là một tập con khác rỗng của một không gian vector topo Hausdorff $Z$ cho trước. Điều này có nghĩa là bài toán (VEP) được nhúng vào họ các bài toán sau, với mỗi $\lambda \in \Lambda$,

$(\text { VEP })_{\lambda}$ Tìm $\bar{x} \in K(\lambda)$ sao cho $f(\bar{x}, y, \lambda) \notin$ $-C \backslash\left\{\mathbf{0}_{Y}\right\}$ với mọi $y \in K(\lambda)$.

Với mỗi $\lambda \in \Lambda$, ta kí hiệu tập nghiệm hữu hiệu yếu của $(\operatorname{VEP})_{\lambda}$ là $S(\lambda)$, tức là:

$$
\begin{gathered}
S(\lambda):=\{x \in K(\lambda): f(x, y, \lambda) \notin-\operatorname{core}(C), \forall y \\
\in K(\lambda)\} .
\end{gathered}
$$

Mục tiêu của mục này là nghiên cứu tính nửa liên tục trên của ánh xạ đa trị $S: \Lambda \rightrightarrows X$. Trước hết, ta nhắc lại một số khái niệm về tính nửa liên tục của ánh xạ đa trị và các tính chất có liên quan.

Định nghĩa 4.1 (Aubin \& Frankowska, 1990, Định nghĩa 1.4.1, 1.4.2, Tr. 38) Cho ánh xạ đa trị $Q: \Lambda \rightrightarrows X$ và $\lambda_{0} \in \Lambda$. Khi đó:

- $Q$ được gọi là nửa liên tục trên tại $\lambda_{0}$ nếu với mọi lân cận $U$ của $Q\left(\lambda_{0}\right)$ thì tồn tại một lân cận $N$ của $\lambda_{0}$ sao cho $Q(\lambda) \subset U$ với mọi $\lambda \in U$.

- $Q$ được gọi là nửa liên tục dưới tại $\lambda_{0}$ nếu cho mọi lưới $\left\{\lambda_{\alpha}\right\}$ hội tụ về $\lambda_{0}$ và mọi $x_{0} \in Q\left(\lambda_{0}\right)$ thì tồn tại một lưới $\left\{x_{\alpha}\right\}$ trong $Q\left(\lambda_{\alpha}\right)$ thỏa mãn $x_{\alpha} \rightarrow x_{0}$.

- $Q$ được gọi là liên tục tại $\lambda_{0}$ nếu $Q$ vừa nửa liên tục trên vừa nửa liên tục dưới tại $\lambda_{0}$.

Ta nói rằng ánh xạ $Q$ là nửa liên tục trên (nửa liên tục dưới, liên tục) trên một tập con nào đó nếu nó là nửa liên tục trên (nửa liên tục dưới, liên tục) tại mọi điểm trên tập con đó.

Bổ đề 4.1 (Hu \& Papageorgiou, 1997, Mệnh đề 2.19 , Tr. 41) Cho $Q\left(\lambda_{0}\right)$ là một tập compact. Khi đó, $Q$ là nửa liên tục trên tại $\lambda_{0}$ nếu và chỉ nếu với bất kỳ lưới $\left\{\lambda_{\alpha}\right\}$ hội tụ về $\lambda_{0}$ và $x_{\alpha} \in Q\left(\lambda_{\alpha}\right)$ thì tồn tại một lưới con $\left\{x_{\beta}\right\}$ của $\left\{x_{\alpha}\right\}$ hội tụ về một điểm $x_{0}$ nào đó thuộc $Q\left(\lambda_{0}\right)$.

Bổ đề 4.2 (Hu \& Papageorgiou, 1997, Mệnh đề 2.6, Tr. 37) Ánh xạ đa trị $Q: \Lambda \rightrightarrows X$ là nửa liên tục dưới tại $\lambda_{0}$ nếu cho mọi lưới $\left\{\lambda_{\alpha}\right\}$ hội tụ về $\lambda_{0}$ thì $Q\left(\lambda_{0}\right) \subset \liminf Q\left(\lambda_{\alpha}\right)$, với

$\liminf Q\left(\lambda_{\alpha}\right):=\left\{x_{0} \in X: \exists x_{\alpha} \in Q\left(\lambda_{\alpha}\right), x_{\alpha} \rightarrow x_{0}\right\}$.

Định lý 4.1

Giả sử các điều kiện sau đây được thỏa mãn:

(i) ánh xạ $K$ là liên tục và có giá trị compact trên $\Lambda$

(ii) với mọi $x \in K, f(x, x, \lambda) \nless \mathbf{0}_{Y}$;

(iii) với mọi $x \in K$, tập $\{y \in K: f(x, y, \lambda)<$ $\mathbf{0}_{Y}$ \} là một tập lồi;

(iv) với mọi $y \in K, f$ là $C$-nửa liên tục trên trên $K \times K \times \Lambda$.

Khi đó, tập nghiệm $S(\lambda)$ là khác rỗng với mọi $\lambda \in \Lambda$. Đồng thời, ánh xạ nghiệm $S$ là nửa liên tục trên và có giá trị compact trên $\Lambda$. 


\section{Chứng minh:}

Ta thấy rằng các giả thiết của Định lý 3.1 được thỏa mãn nên ta suy ra $S(\lambda)$ khác rỗng với mọi $\lambda \in$ $\Lambda$.

Ta chứng minh ánh xạ nghiệm $S$ là nửa liên tục trên trên $\Lambda$. Lấy bất kỳ $\lambda_{0} \in \Lambda$, ta sẽ chứng minh $S$ là nửa liên tục trên tại $\lambda_{0}$. Giả sử ngược lại rằng $S$ không là nửa liên tục trên tại $\lambda_{0}$. Khi đó, ta tìm được một lân cận mở $U$ của $S\left(\lambda_{0}\right)$, một lưới $\left\{\lambda_{\alpha}\right\}$ hội tụ về $\lambda_{0}$ và $x_{\alpha} \in S\left(\lambda_{\alpha}\right)$ sao cho $x_{\alpha} \notin U$ với mọi $\alpha$. Do tính compact và tính nửa liên tục trên của $K$ nên theo Bổ đề 4.1 ta có thể giả sử rằng $x_{\alpha} \rightarrow x_{0}$ với $x_{0} \in$ $K\left(\lambda_{0}\right)$. Nếu $x_{0} \notin S\left(\lambda_{0}\right)$ thì tồn tại $y_{0} \in K\left(\lambda_{0}\right)$ sao cho $f\left(x_{0}, y_{0}, \lambda_{0}\right)<\mathbf{0}_{Y}$. Do $y_{0} \in K\left(\lambda_{0}\right)$ và $K$ là nửa liên tục dưới tại $\lambda_{0}$ nên theo Định nghĩa 4.1(b) thì tồn tại một lưới $\left\{y_{\alpha}\right\}$ trong $K\left(\lambda_{\alpha}\right)$ sao cho $y_{\alpha} \rightarrow y_{0}$. Vì $x_{\alpha} \in S\left(\lambda_{\alpha}\right)$ nên ta có $f\left(x_{\alpha}, y_{\alpha}, \lambda_{\alpha}\right) \nless \mathbf{0}_{Y}$ với mọi $\alpha$. Từ tính $C$-nửa liên tục của $f$ trong giả thiết (iv) và $f\left(x_{0}, y_{0}, \lambda_{0}\right)<\mathbf{0}_{Y}$, ta suy ra tồn tại một lân cận $U_{0}$ của $\left(x_{0}, y_{0}, \lambda_{0}\right)$ sao cho $f(x, y, \lambda)<\mathbf{0}_{Y}$ với mọi $(x, y, \lambda) \in U_{0}$. Do $\left(x_{\alpha}, y_{\alpha}, \lambda_{\alpha}\right) \rightarrow\left(x_{0}, y_{0}, \lambda_{0}\right)$ nên tồn tại $\alpha_{0}$ sao cho $\left(x_{\alpha}, y_{\alpha}, \lambda_{\alpha}\right) \in U_{0}$ với $\alpha \geq \alpha_{0}$. Khi đó, $f\left(x_{\alpha}, y_{\alpha}, \lambda_{\alpha}\right)<\mathbf{0}_{Y}$ với $\alpha \geq \alpha_{0}$. Ta có điều mâu thuẫn. Do đó, $x_{0} \in S\left(\lambda_{0}\right) \subset U$. Đây lại là một không thể vì $x_{\alpha} \notin U$ với mọi $\alpha$.

Bây giờ ta chứng minh $S(\lambda)$ là một tập compact. Lấy bất kỳ $x_{\alpha} \in S(\lambda)$ với $x_{\alpha} \rightarrow x_{0}$. Khi đó, với mọi $y \in K(\lambda)$, ta có $f\left(x_{\alpha}, y, \lambda\right) \nless \mathbf{0}_{Y}$. Lập luận tương tự như trên, ta cũng chứng minh được $x_{0} \in S(\lambda)$. Do đó, $S(\lambda)$ là tập đóng trong $K(\lambda)$. Mà $K(\lambda)$ là một tập compact nên ta suy ra $S(\lambda)$ cũng là một tập compact.

\section{KẾT LUẬN}

Trong bài báo này, bài toán cân bằng vector theo nón thứ tự có phần trong đại số khác rỗng đã được nghiên cứu. Trước hết, các điều kiện đủ cho bài toán có nghiệm hữu hiệu yếu đã được thiết lập dựa vào bổ đề KKM-Fan (Định lý 3.1). Sau đó, tính nửa liên tục trên của ánh xạ nghiệm khi dữ liệu bài toán bị nhiễu bởi tham số cũng được khảo sát (Định lý 4.1). Các kết quả đạt được trong bài báo là mới. Cách tiếp cận này sẽ đạt được nhiều kết quả mới hơn nữa về điều kiện tồn tại nghiệm, điều kiện ổn định nghiệm cũng như các chủ đề khác cho các lớp bài toán trong tối ưu mà không gian ảnh không có trang bị cấu trúc topo.

\section{TÀI LIỆU THAM KHẢO}

Aliprantis, C.D., \& Border, K.C. (2007). Infinite Dimensional Analysis: A Hitchhiker's Guide, Third Edition. Springer. Berlin.
Anh, L.Q., Duoc, P.T., \& Tam, T.N. (2018). On Hölder continuity of solution maps to parametric vector primal and dual equilibrium problems. Optimization, 67(8), 1169-1182.

Anh, L.Q., Duoc, P.T., \& Tam, T.N., 2020. On the stability of approximate solutions to set-valued equilibrium problems. Optimization, 69(7-8), 1583-1599.

Ansari, Q. H., Oettli, W., \& Schlager, D. (1997). A generalization of vectorial equilibria. Mathematical Methods of Operations ResearchZOR, 46(2), 147-152.

Anh, L.Q., Duoc, P.T., Tam, T.N., \& Thang, N.C. (2021). Stability analysis for set-valued equilibrium problems with applications to Browder variational inclusions. Optimization Letters, 15(2), 613-626.

Anh, L.Q., Khanh, P.Q., \& Tam, T.N. (2019). Continuity of approximate solution maps of primal and dual vector equilibrium problems. Optimization Letters, 13(1), 201-211.

Ansari, Q.H. (2000). Vector equilibrium problems and vector variational inequalities. In: F. Giannessi

(Ed.), Vector Variational Inequalities and Vector Equilibria (pp. 1-14). Kluwer Academic. Dordrecht.

Ansari, Q.H., Konnov, I.V., \& Yao, J.C. (2001). Existence of a solution and variational principles for vector equilibrium problems. Journal of Optimization Theory and Applications, 110(3), 481-492.

Ansari, Q.H. (2008). Existence of solutions of systems of generalized implicit vector quasiequilibrium problems. Journal of Mathematical Analysis and Applications, 341(2), 1271-1283.

Aubin, J.P., \& Frankowska, H. (1990). Set-Valued Analysis. Birkhäuser. Boston.

Bianchi, M., Hadjisavvas, N., \& Schaible, S. (1997). Vector equilibrium problems with generalized monotone bifunctions. Journal of Optimization Theory and Applications, 92(3), 527-542.

Bigi, G., Adela, C., \& Kassay, G. (2012). Existence results for strong vector equilibrium problems and their applications. Optimization, 61(5), 567-583.

Bonnans, J.F., \& Shapiro, A. (2000). Perturbation Analysis of Optimization Problems. Springer. New York.

Chen, G.Y., Huang, X.X., \& Yang, X.Q., (2005). Vector Optimization: Set-Valued and Variational Analysis. Springer. Berlin.

Fan, K. (1961). A generalization of Tychonoff's fixed point theorem. Mathematische Annalen, 142(3), 305-310. 
Gong, X.H. (2006). Strong vector equilibrium problems. Journal of Global Optimization, 36(3), 339-349.

Hu, S., \& Papageorgiou, N. (1997). Handbook of Multivalued Analysis, Volume I: Theory. Kluwer. Boston.

Huang, N.J., Li, J., \& Yao, J.C. (2007). Gap functions and existence of solutions to a system of vector equilibrium problems. Journal of
Optimization Theory and Applications, 133(2), 201-212.

Kantorovitz, S. (2003). Introduction to Modern Analysis. Oxford University Press. Oxford.

Oettli, W. (1997). A remark on vector-valued equilibria and generalized monotonicity. Acta Mathematica Vietnamica, 22(1), 213-221.

Tam, T.N. (2021). On Hölder continuity of solution maps to parametric vector Ky Fan inequalities. TOP. https://doi.org/10.1007/s11750-021-00602-4 